hep-ph/9801444

June 17, 1998

\title{
Study of the Uncertainty of the Gluon Distribution
}

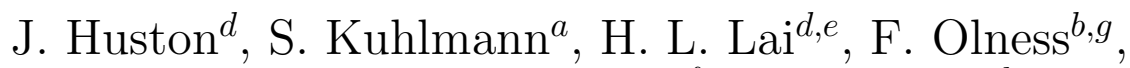

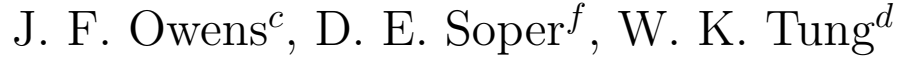 \\ ${ }^{a}$ Argonne National Laboratory, ${ }^{b}$ Fermi National Accelerator Laboratory, \\ ${ }^{c}$ Florida State University, ${ }^{d}$ Michigan State University, \\ ${ }^{e}$ National Tsing Hua University, ${ }^{f}$ University of Oregon, \\ ${ }^{g}$ Southern Methodist University
}

\begin{abstract}
The uncertainty in the calculation of many important new processes at the Tevatron and LHC is dominated by that concerning the gluon distribution function. We investigate the uncertainty in the gluon distribution of the proton by systematically varying the gluon parameters in the global QCD analysis of parton distributions. The results depend critically on the parton momentum fraction $x$ and the QCD scale $Q^{2}$. The uncertainties are presented for integrated gluon-gluon and gluon-quark luminosities for both the Tevatron and LHC as a function of $\sqrt{\tau}=\sqrt{x_{1} x_{2}}=\sqrt{\hat{s} / s}$, the most relevant quantity for new particle production. The uncertainties are reasonably small, except for large $x$.
\end{abstract}

\footnotetext{
${ }^{*}$ This work was supported in part by the DOE and NSF.
} 
Many hadron-collider signatures of physics beyond the Standard Model have a gluon in the initial state, in either the signal process or the important background processes. Other new signatures within the Standard Model can also have gluons in the initial state. One example of this is the production of a light Higgs boson at the LHC via the process $g g \rightarrow$ $H \rightarrow \gamma \gamma$. Another example is the measurement of $V_{t b}$ using single-top production at the Tevatron via the process $g W \rightarrow t b$. It is important to estimate the theoretical uncertainty in the Quantum Chromodynamics (QCD) calculations of these new processes. Since the quark distributions of the nucleon are relatively well-determined, the dominant uncertainty in these cases is due to that of the gluon distribution. The conventional method of estimating parton distribution uncertainties is to compare different published parton parameterizations. This is a completely unreliable approach since the authors of most published sets of parton distributions adopt similar assumptions and use similar data sets. The differences between these sets have little to do with the range of possible variations of the parton distributions as constrained by current theory and available data. In this paper we focus on the uncertainty of the gluon distribution within the framework of the CTEQ global QCD analysis [1], and present a more complete estimate of the uncertainties. Not surprisingly, we will find that the uncertainty is a function of the gluon $x$ and $Q^{2}$.

Ideally, one might hope to perform a full error analysis and provide an error-correlation matrix for all the parton distributions. This ambitious goal is, however, impractical at this time for two reasons. First, only a subset of available experiments provide correlation information for their data sets in a way suitable for this analysis. Secondly, there is no established way to quantify the theoretical uncertainties for the diverse physical processes used in the global analysis. One possibility, explored in reference [2], is to invoke only the deep-inelastic scattering (DIS) process, to use the DIS data sets with the needed correlation information, and only use those data points at high $Q^{2}$ where the theoretical uncertainties are expected to be negligible. While this procedure is of methodological interest, it leaves out many useful data sets and the uncertainties obtained for the gluon are clearly unrealistic.

The approach we adopt in this paper is to systematically vary the gluon distribution parameters in the global analysis framework. We then conservatively delineate the range of admissible distributions as that bounded by fits which show clear disagreements with more than one data set. For this purpose, we adopt the CTEQ4M parton distribution set [1] as the standard and explore the range of possible variations of the gluon distribution around it. The conclusions of this study should apply to all modern parton distribution sets since they are in rather good agreement with each other. [1, []

Constraints Based on the Momentum Sum Rule The momentum fraction of the proton carried by quarks is determined by deep-inelastic scattering data to be $58 \%$ in the CTEQ4M analysis $(\mathrm{Q}=1.6 \mathrm{GeV})$ [1]. The uncertainty in this number is mainly due to normalization uncertainties of the experimental data sets, which is typically $\pm 2 \%$. Therefore the total gluon momentum fraction in the CTEQ4M fit is $42 \%$ with an uncertainty of about $2 \%$. This is an extremely important constraint that is not fully appreciated. If the flux of gluons in a certain $x$ range is increased, the flux must be reduced by almost the same amount somewhere else.

Table 1 shows how the momentum fraction of gluons within the proton is distributed as 


\begin{tabular}{|c|c|}
\hline $\mathrm{x}$ Bin & Momentum Fraction \\
\hline $10^{-4}$ to $10^{-3}$ & $0.6 \%$ \\
$10^{-3}$ to 0.01 & $3 \%$ \\
0.01 to 0.1 & $16 \%$ \\
0.1 to 0.2 & $10 \%$ \\
0.2 to 0.3 & $6 \%$ \\
0.3 to 0.5 & $5 \%$ \\
0.5 to 1.0 & $1 \%$ \\
\hline
\end{tabular}

Table 1: The fraction of proton momentum carried by gluons in different $x$ bins. This is for the CTEQ4M parameterization at $\mathrm{Q}=1.6 \mathrm{GeV}$.

a function of $x$ for the CTEQ4M parameterization at $\mathrm{Q}=1.6 \mathrm{GeV}$. The largest component of the gluon momentum is carried at medium values of $x$, since this has the largest product of the number of gluons and the momentum fraction of each gluon. A simple exercise using these momentum fractions is illustrative. If we assume the flux of gluons in the range $0.01<x<0.3$ is decreased by $20 \%$ below the CTEQ4M value, what happens to the momentum sum rule constraints? From Table 1, 32\% of the proton momentum is in this $x$ region, a $20 \%$ decrease is $6.4 \%$ which is the amount that has to be increased elsewhere. The compensation would have to come from a $\times 2.8$ increase $((6.4+3.6) / 3.6)$ in gluons below $x=0.01$, or a $\times 2.1$ increase $((6.4+6) / 6)$ in gluons above $x=0.3$, or a combination of the two. Typical uncertainties from HERA (at $\mathrm{Q}=1.6 \mathrm{GeV}$ ) in the gluon distribution for small $x$ are shown to be 30-40\% [4], therefore not much compensation can come from small $x$. At larger $x$ the fixed target Drell-Yan data is sensitive to the gluon since the sea quarks couple to the gluons. As shown in the next section, the increase in the gluon distribution described above would increase the predicted Drell-Yan cross sections at large $x$ by $>40 \%$, ruining the present agreement with CTEQ4M parton distributions. Therefore the needed compensation for the $20 \%$ change in gluons is unlikely to come from $x>0.3$ either. This exercise illustrates the important constraint on the gluon distribution at medium $x$ from the momentum sum rule, and also serves to explain the quantitative results on parton distribution uncertainties discussed in the following sections. Naturally this exercise is simplified since the momentum fraction of the gluon changes with $Q^{2}$. In Fig. 11 we plot the gluon momentum fraction distribution for $\mathrm{Q}=5 \mathrm{GeV}$ and for $\mathrm{Q}=100 \mathrm{GeV}$. In this plot the area under the curve in any $x$ interval is the gluon momentum fraction in that region. The evolution to smaller parton $x$ as Q increases is evident, but in both cases the bulk of the gluon momentum is at medium values of $x$.

Scanning the Gluon Parameters We now perform a detailed study of the range of possible variation of the gluon distribution by systematically varying the gluon parameters in a global analysis. The CTEQ4 gluon parametrization is: $A_{0} x^{A_{1}}(1-x)^{A_{2}}\left(1+A_{3} x^{A_{4}}\right)$, with $A_{0}=1.1229, A_{1}=-0.206, A_{2}=4.673, A_{3}=4.269, A_{4}=1.508$ for the standard CTEQ4M parton distribution set. We begin by fixing $\alpha_{S}$ to be the CTEQ4M value $\left(\alpha_{S}\left(M_{Z}\right)=0.116\right)$, 
which is close to the current world average of $0.118 \pm 0.003$. [5] We have studied the variation of the gluon with $\alpha_{S}$ in a previous publication [1], and will discuss this more later. We have then systematically varied the values of $A_{1}, A_{2}$, and $A_{3}$, each time refitting the other quark and gluon parameters using the CTEQ procedure described in Ref. [1]. The range of variation of each parameter was expanded until clear disagreements with more than one data set were observed. In order to be conservative in this study, we performed these scans using only the well-established DIS and Drell-Yan data sets. This also allows us to establish a baseline uncertainty estimate with the processes that are best understood theoretically. We will discuss the possible impact of direct photon and jet production data in a later section.

As an example of one of these parameter scans, the total $\chi^{2}$ from the $A_{2}$ scan is shown in Fig. 2. The parameter $A_{2}$ is the exponent of the $(1-x)$ factor. It is varied over the wide range from 1.0 to 9.0, with the CTEQ4M value being 4.673. The total $\chi^{2}$ variable was only used for guidance in determining acceptable gluon distributions, a strict cut was not applied. In practice we examined closely every data set for every variation, and coupled with our experience with experimental and theoretical uncertainties, determined which gluon distributions caused disagreements with data that could not be explained by such uncertainties. The four worst fits in Fig. 2 have examples of clear disagreements with some data sets. Figure 3 shows the change in the gluon distribution, and the corresponding effect on three data sets for the $A_{2}=1.0$ fit. This change in gluon distribution is almost exactly that described earlier concerning the momentum sum rule, the upper left figure demonstrates this. In another example, the $A_{2}=9.0$ variation, all of the fixed target DIS data sets (with 60-170 data points each) had increased $\chi^{2}$ of 15 units or more over those of the CTEQ4M fit. Similar criteria were applied for extreme fits to the $A_{1}, A_{3}$ parameter scans. Fig. 1 shows the ratio of the gluon distributions from these extreme fits to CTEQ4M, with $\mathrm{Q}=5 \mathrm{GeV}$ on top and $\mathrm{Q}=100 \mathrm{GeV}$ on bottom. The effect of the momentum sum rule constraint is once again dramatically demonstrated at $\mathrm{Q}=5 \mathrm{GeV}$. The relatively small changes at moderate values of $x$ are compensated by large changes at small and large $x$. The range of gluon distributions at $\mathrm{Q}=100 \mathrm{GeV}$ is interesting since the smallest invariant mass for typically new particle production is around $\mathrm{M}=100 \mathrm{GeV}$. Notice that the variation of the gluon is only $\pm 15 \%$ below $x=0.15$ at this scale; and these are the fits which already show clear conflicts with existing data sets, some of which cause increases in $\chi^{2}$ of more than 200 units. This demonstrates how QCD evolution tends to wash out the differences in parton distributions at low $Q$. At larger $x$ the differences between the gluon variations remain fairly constant as $Q$ increases. This is because of the influence of even larger differences in the gluon distribution for $x>0.5$ (not shown on the plot) that are feeding down into the $x=0.2-0.5$ region at higher $Q^{2}$.

A reasonable estimate of the current uncertainties on the gluon distribution can be obtained by examining the range of variations spanned by those fits which do not clearly contradict any of the data sets used. Fig. 5 shows the result obtained from all such scans. The pattern seen is similar to that shown in Fig. 4. At $Q=100 \mathrm{GeV}$, the range of variation for the gluon is relatively small below $x<0.15$; it increases steadily as $x$ increases. Below $x<0.15$ the range of gluons appears to be of the order $7 \%$. This may not yet be the true range of gluon distributions since a fixed value of $\alpha_{S}$ has been used in this study, whereas

${ }^{1}$ We found the variation of $A_{3}$ was easily compensated by changes in $A_{4}$, and vice versa. Therefore in the $A_{3}$ parameter scan described below $A_{4}$ was fixed to the CTEQ4M value. 
$\alpha_{S}$ and $G(x)$ are known to be correlated in the global analysis. It is useful to decouple the two effects since $\alpha_{S}$ can be measured in a variety of ways independent of parton distributions, and these measurements are continuing to improve. At present the PDG value of $\alpha_{S}$ is $0.118 \pm 0.003$, a $2.5 \%$ uncertainty. We have varied $\alpha_{S}$ by $8 \%$, between 0.113 and 0.122 , and found a $3 \%$ variation in the gluon distribution below $x<0.15$ at $\mathrm{Q}=100 \mathrm{GeV}$, this sets the scale for the additional uncertainty in the gluon distribution due to $\alpha_{S}$ variations. In addition, we have taken the correlation between $\alpha_{S}$ and the gluon distribution into account by refitting the previous extreme variations that caused conflicts with present data sets, this time allowing $\alpha_{S}$ to vary. The general conclusions remain the same as that stated before, with a slight increase in the magnitude of the uncertainty: the range of gluon distributions is within $10 \%$ of CTEQ4M below $x<0.15$ and $Q>100 \mathrm{GeV}$, and the uncertainties grow significantly at larger $x$.

One concern is that the relatively small range of variation on the gluon distribution may be an artifact of a too-restrictive parameterization, coupled with the constraints of the momentum sum rule. To answer this question, we have modified the gluon distribution parameterization according to the following ansatz:

$$
x G(x)=x G^{\mathrm{CTEQ} 4}(x)+A_{5}(1-x)^{5}(x-1 / 7) .
$$

The added term affects mainly the shape of the gluon distribution at medium $x$. It is small compared to the standard CTEQ4M contribution for $x \ll 1$ and $x \rightarrow 1$. Furthermore, the total integrated momentum fraction contributed by this term is zero, so that the gluon distribution is not forced to change at small or large $x$ in order to maintain the momentum sum rule. We assessed possibilities for modifying the gluon distribution at medium $x$ by varying values for $A_{5}$. As in the previous parameter scan, for each value of $A_{5}$ we refit the other gluon and quark parameters. In this case we fixed the gluon's $A_{3}$ and $A_{4}$ to the CTEQ4M value, since these parameters have a similar effect at medium $x$ to that of $A_{5}$. We then varied $A_{5}$ until clear disagreements with some data sets arose. The fits resulting from this study gave rise to gluon distributions entirely within the bands shown in Fig. 5. Thus, we are confident that the quoted uncertainties are not an artifact of the gluon distribution parameterization.

Uncertainty on the gluon-gluon luminosity function For assessing the range of predictions on cross-sections for Standard Model and new physics processes, it is more important to know the uncertainties on the gluon-gluon and gluon-quark luminosity functions at the appropriate kinematic region (in $\tau=x_{1} x_{2}=\hat{s} / s$ ), rather than on the parton distributions themselves. [6] Therefore, we turn to the relevant integrated parton-parton luminosity functions. The gluon-gluon luminosity function is defined to be:

$$
\tau d L / d \tau=\int_{\tau}^{1} G\left(x, Q^{2}\right) G\left(\tau / x, Q^{2}\right) d x / x .
$$

This quantity is directly proportional to the cross-section for the s-channel production of a single particle; it also gives a good estimate for more complicated production mechanisms. It is most appropriate when the experimental acceptances do not play a major role in the cross section calculation. We have calculated the gluon-gluon luminosity function for the 
parton distribution variations shown in Fig. 5. This was done for both the Tevatron and LHC energies.

Figure 6 shows the ratio of the gluon-gluon luminosity normalized to the corresponding result from CTEQ4M, for the variations discussed in the last section, as a function of $\sqrt{\tau}$. Here we take $Q^{2}=\tau s$, which naturally takes the $Q^{2}$ dependence of the gluon distribution into account as one changes $\sqrt{\tau}$. This choice of scale parameter is a common choice in a lowest order calculation. The bands are cutoff below $Q=25 \mathrm{GeV}$, since this region is almost certainly irrelevant for new particle production at the Tevatron or LHC. The top figure is for the $\operatorname{LHC}(\sqrt{s}=14 \mathrm{TeV})$, and the bottom figure is for the Tevatron $(\sqrt{s}=2 \mathrm{TeV})$. The region of production of a 100-140 GeV Higgs at the LHC is indicated, it lies in the medium $x$, large $Q^{2}$ region where the range of variation is $10 \%$. The size of the bands for $x>0.1$ has now grown since we are squaring the variations seen in Fig. 5. This emphasizes the need for much more quality information about the parton distributions at large $x$ than is available from DIS+Drell-Yan data sets used in this analysis.

Uncertainty on the gluon-quark luminosity function In analogy with the discussion of gluon-gluon luminosities in the last section, we now study the variations of gluon-quark luminosities. Figure 0 shows the ratio of the gluon-quark luminosity normalized to the corresponding result from CTEQ4M, for the same variations used in the last section, as a function of $\sqrt{\tau}$. Indicated on the figure is the region of single-top production at the Tevatron, near $\sqrt{\tau}=0.1$. The variations indicated by the solid curves do not include the uncertainty of the quark distributions, except those of the sea quarks driven by different gluon variations. Since this is the flavor-independent sum of quarks, which is generally constrained to within $2-3 \%$ by DIS data, we expect these uncertainties to be negligible. There is one exception, however, and that is at very large $x$ and $Q^{2}$, which is discussed in reference [7]. The toy model in that paper provides more quarks for $x>0.5$ at large $Q^{2}$ than present in CTEQ4M, and is indicated by the dotted curve in Fig. 17. This model is not meant to be taken seriously as the true set of parton distributions in nature, but should be indicative of the current uncertainty in the quark distributions in this kinematic region. The dotted curve is only significant at very large $\sqrt{\tau}$, but emphasizes once again the need for much more quality information about parton distributions at large $x$.

Summary of gluon-gluon and gluon-quark uncertainties We summarize in Table 2 what one observes in the last two sections. Since the sizes of the bands were almost identical for $\sqrt{s}=2$ or $14 \mathrm{TeV}$, we only give one set of numbers for the gluon-gluon uncertainty and one set for gluon-quark. Above $\sqrt{\tau}>0.4$ the uncertainties for both gluon-gluon and gluon-quark are increasing rapidly and should simply be considered as being unconstrained with these data. These are not meant to be precise uncertainties obtained by rigorous statistical analysis, but reasonable error estimates in the same spirit as the estimate for the uncertainty in the theory that one obtains by varying the $\mu^{2}$ scale in the calculation. They are also subjective to the extent that they depend on the choices of what constitute acceptable global fits. However, we showed that even if one allows fits with total $\chi^{2}$ more than 200 units (for 1300 data points) greater than that of CTEQ4M, the range of gluon distributions for $x<0.15$ is only $5 \%$ larger than is shown in this table. We therefore consider this a robust 
and conservative estimate of the uncertainties due to the parton distributions.

\begin{tabular}{|c|c|c|}
\hline$\sqrt{\tau}$ Range & Gluon-Gluon & Gluon-Quark \\
\hline$<0.1$ & $\pm 10 \%$ & $\pm 10 \%$ \\
$0.1-0.2$ & $\pm 20 \%$ & $\pm 10 \%$ \\
$0.2-0.3$ & $\pm 30 \%$ & $\pm 15 \%$ \\
$0.3-0.4$ & $\pm 60 \%$ & $\pm 20 \%$ \\
\hline
\end{tabular}

Table 2: Recommended uncertainties on gluon-gluon and gluon-quark luminosities for both the Tevatron and LHC, as a function of $\sqrt{\tau}$. This is compared to CTEQ4M as the default parton distribution set.

Comments on Other Data Sets For the analysis presented above, we have only used deep-inelastic scattering and Drell-Yan data. Historically, direct photon production data were thought to place good constraints on the gluon. However, it was pointed out some time ago 8 that the theoretical uncertainty of NLO QCD theory was too large to allow an accurate phenomenological analysis of direct photon data and that the available experimental results showed a clear pattern of deviation from NLO theory expectations. The recent publication of the E706 direct photon data set [9] has dramatically confirmed this observation (the deviation from NLO theory is as large as a factor of 3-4), and provided clear experimental evidence for the need to include initial state $k_{t}$ effects due to multi-gluon radiation, as proposed in Ref. [8]. Thus, theoretical progress on the resummation of multi-gluon radiation is a prerequisite for using direct photon data as a reliable constraint on the gluon distribution.

The original CTEQ4 analysis also included CDF and D0 single jet inclusive data in the global fits. Unfortunately, the experimental situation with these data has become murkier with the more recent D0 data analysis [10]. Even though the CDF and D0 data sets are consistent within the experimental systematic error bands, they are presently inconsistent within statistical uncertainties. Under these circumstances, the proper treatment of the systematics has become important. This awaits final publication of both data sets.

We note that it is unlikely that either the direct photon or high $p_{t}$ jet measurements will be able to reduce significantly the uncertainties for $x<0.15$, since the experimental normalization uncertainties and theoretical scale dependence is typically $10 \%$. The main contribution these processes can give in the future is at large $x$, and in the shape of the gluon distribution at medium values of $x$.

Our baseline variations of the gluon distribution can be used as the benchmark for comparison with present and future measurements which have the potential to narrow the uncertainties. For this purpose the various parton distribution sets which typify the variations shown in this paper will be made available to interested users [11].

Conclusions We have studied the uncertainty in the gluon distribution of the nucleon by systematically varying the relevant parameters in the QCD global analysis. This uncertainty dominates the current uncertainty in the calculation of many important new processes at 
the Tevatron and LHC. The uncertainty depends critically on parton $x$ and $Q^{2}$. We present a table of estimated uncertainties for integrated gluon-gluon and gluon-quark luminosities

for both the Tevatron and LHC as a function of $\sqrt{\tau}=\sqrt{x_{1} x_{2}}=\sqrt{\hat{s} / s}$. The uncertainties are reasonably small, except for large $x$, where future emphasis should be placed.

\section{References}

[1] H.L. Lai et al., Phys. Rev. D55:1280, (1997).

[2] S. Alekhin, hep-ph/9611213, submitted to Elsevier Science

[3] A.D. Martin et al., Phys. Lett. B387:419, (1996).

[4] Proceedings of the 5th International Workshop on Deep Inelastic Scattering and QCD, DIS97, AIP Conference Proceedings No. 407.

[5] Particle Data Group, Phys. Rev. D54:1, (1996)

[6] E. Eichten et al., Rev. Mod. Phys. 56:579, (1984)

[7] S. Kuhlmann et al., Phys. Lett. B409:271, (1997).

[8] J. Huston et al., Phys. Rev D51:6139, (1995)

[9] E706 Collaboration (L. Apanasevich et al.), hep-ex/9711017, FNAL-Pub-97/351-E

[10] D0 Collaboration, "The Dijet Mass Spectrum at D0", International Europhysics Conference on High Energy Physics, August 19-26, 1997, Jerusalem, Israel

[11] All of the eight parton distribution variations described in this paper are available from the CTEQ web site, http://www.phys.psu.edu/ cteq/. 


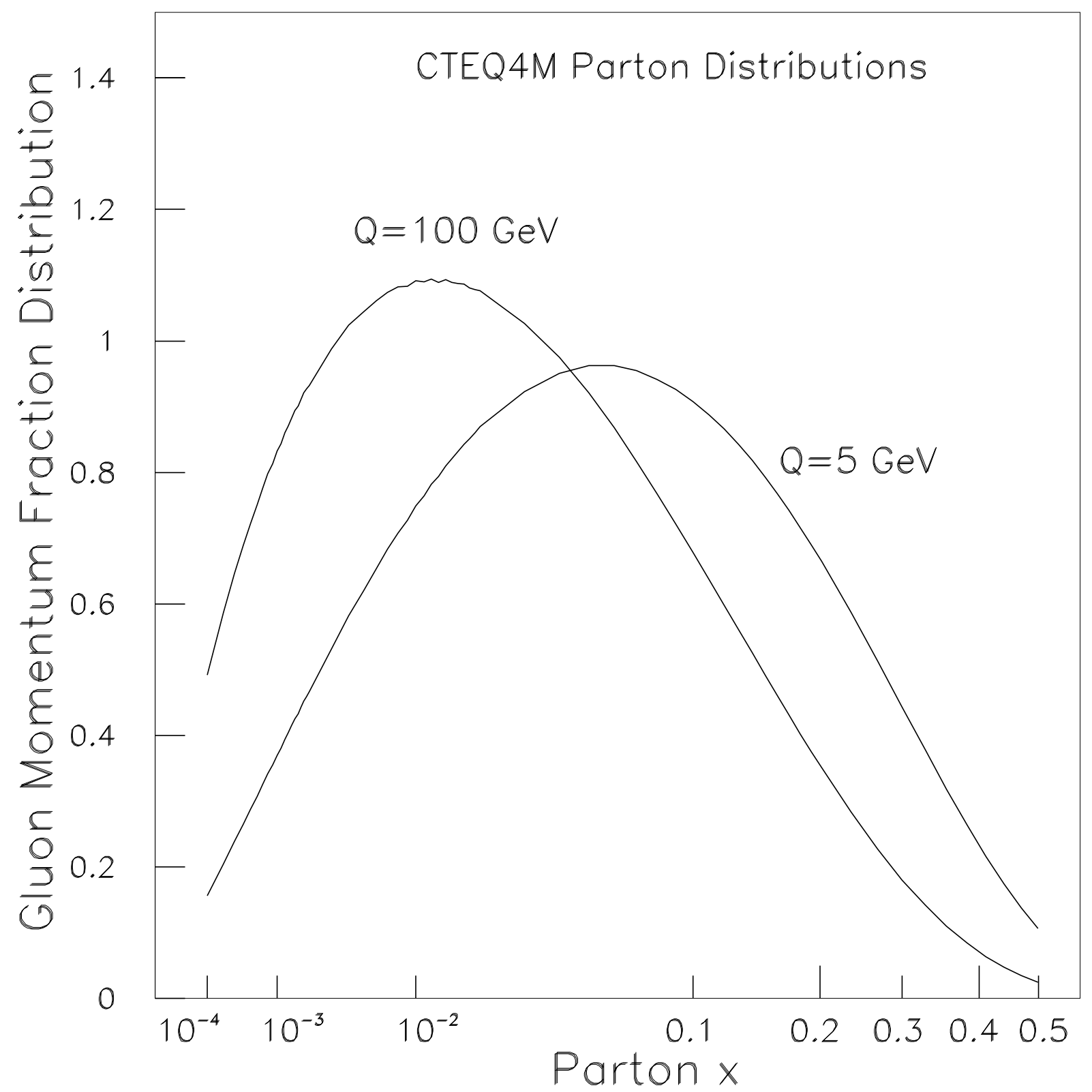

Figure 1: The gluon momentum fraction distribution is shown for $\mathrm{Q}=5 \mathrm{GeV}$ and for $\mathrm{Q}=100$ $\mathrm{GeV}$. 


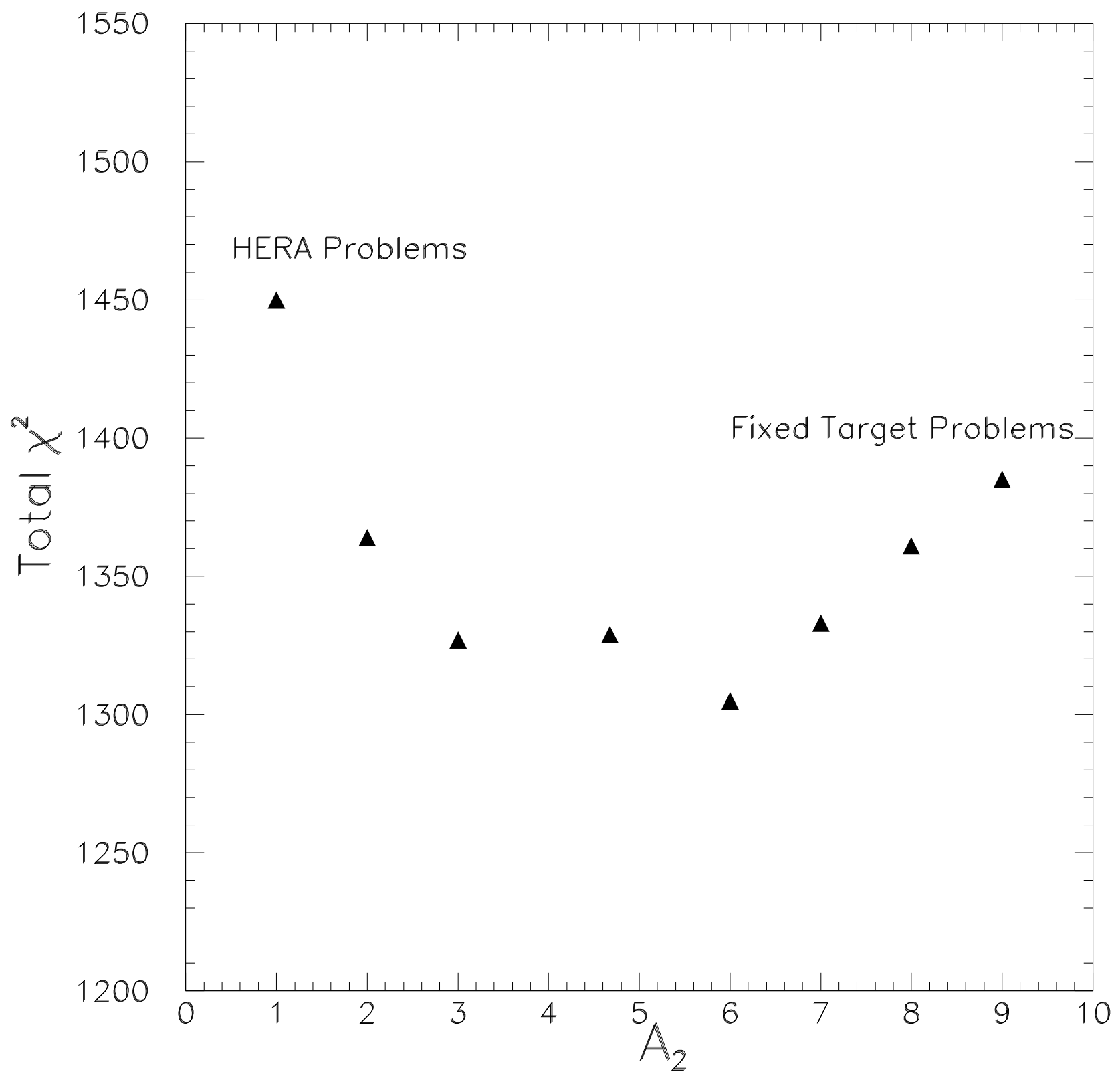

Figure 2: The total $\chi^{2}$ is shown from the $A_{2}$ parameter scan is shown. 

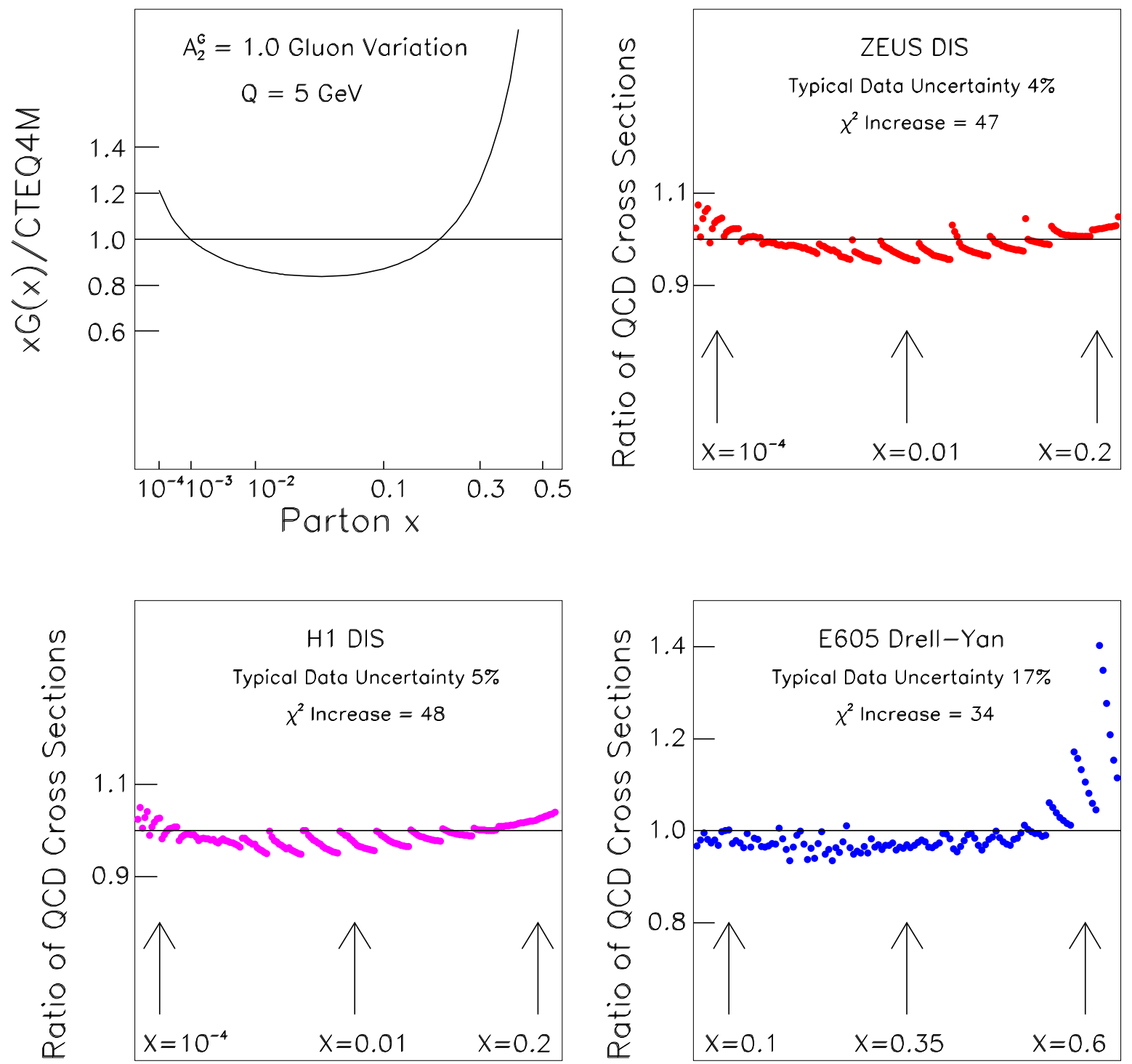

Figure 3: One example of a gluon distribution that causes clear disagreements with data is shown. Upper left is the ratio of the gluon distribution to CTEQ4M. The other three plots show the ratio of QCD predictions for three of the data sets, using the trial gluon distribution. Also indicated on these three plots are the typical data uncertainty, and the change in $\chi^{2}$ for this set of data. 


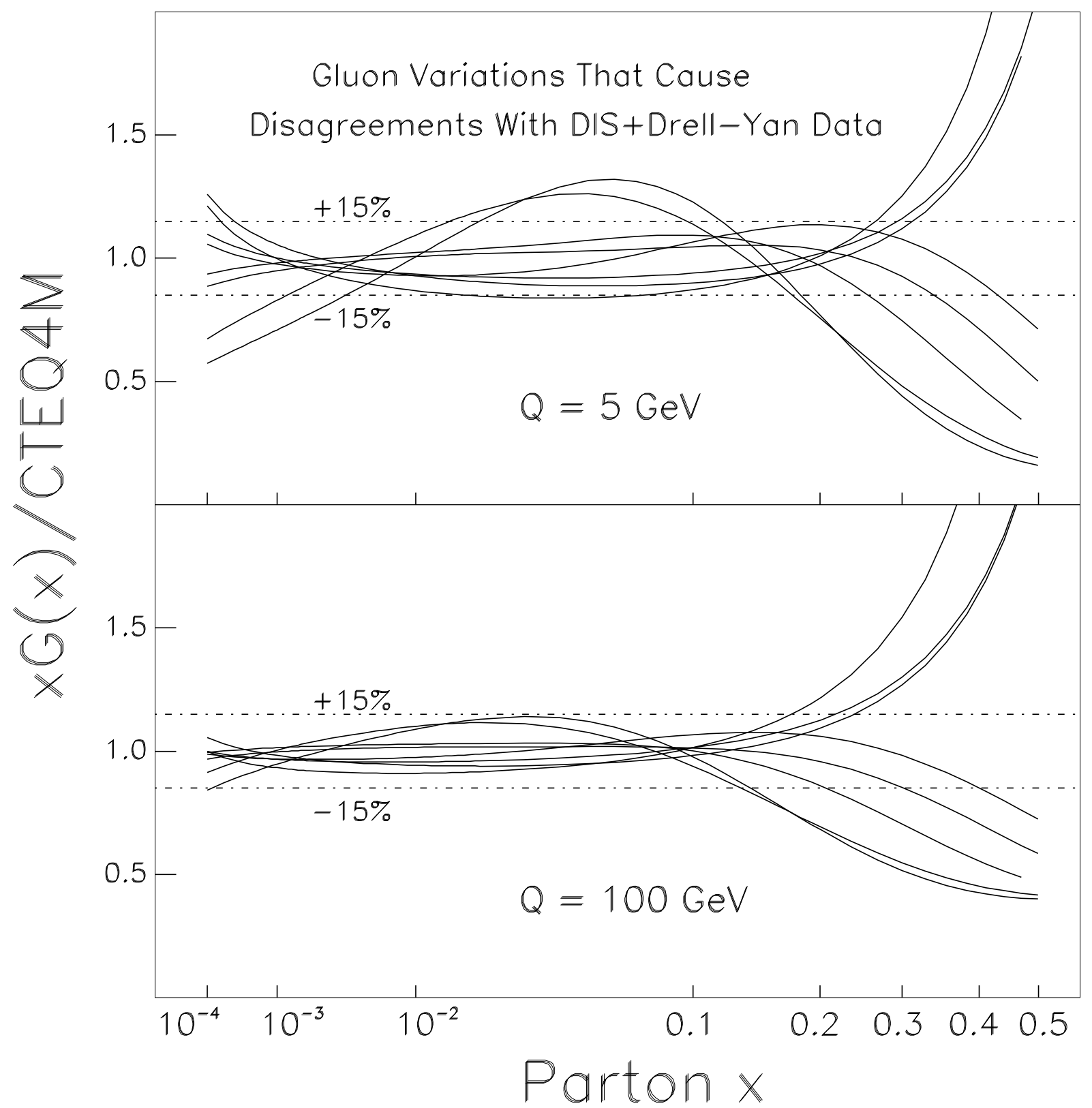

Figure 4: The ratio of gluon distributions compared to CTEQ4M is shown. On top is for $\mathrm{Q}=5$ $\mathrm{GeV}$, and on bottom is $\mathrm{Q}=100 \mathrm{GeV}$. These are the examples that cause clear disagreements with some DIS+Drell-Yan data sets (see text). 


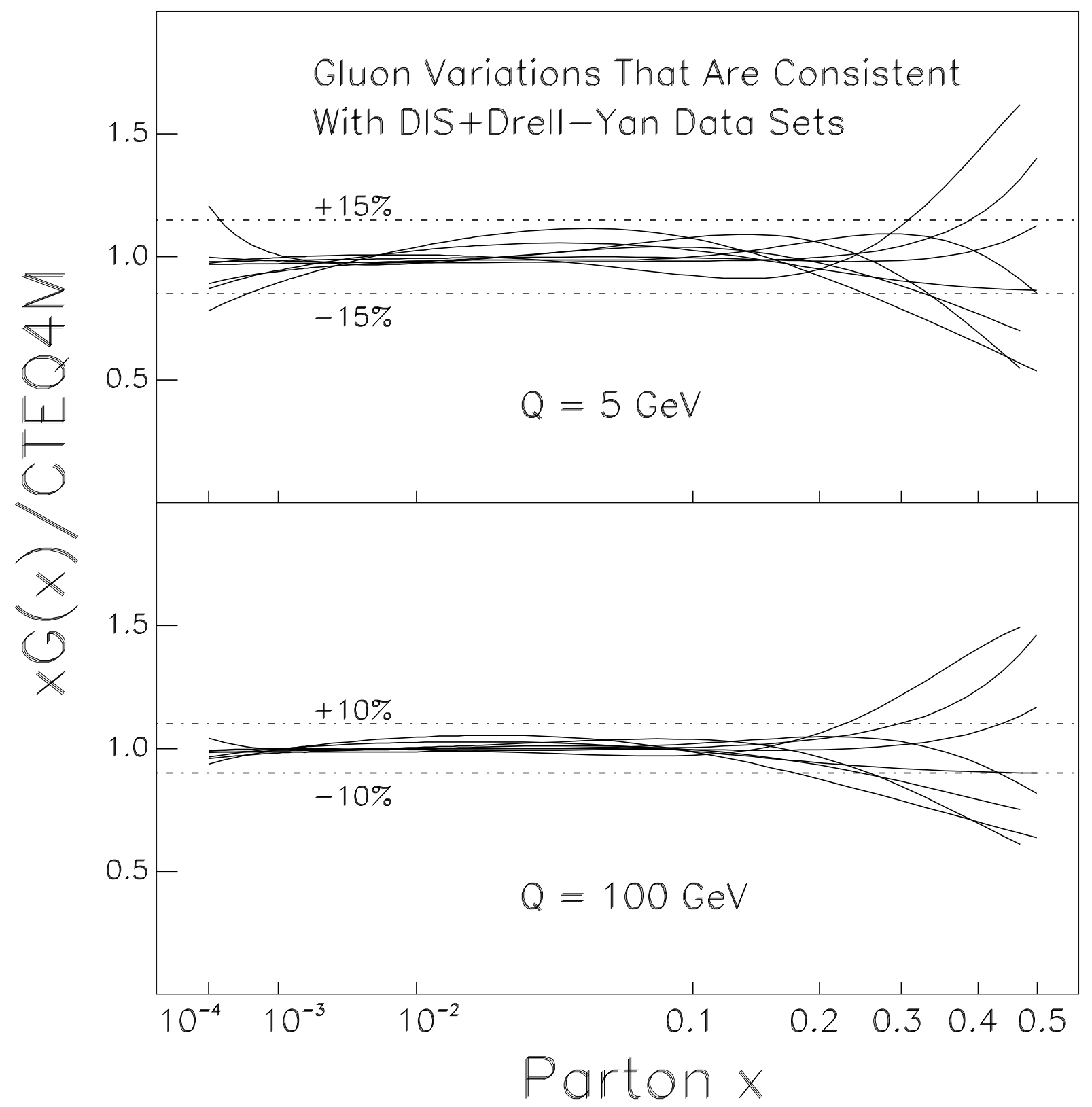

Figure 5: The ratio of gluon distributions compared to CTEQ4M is shown. On top is for $\mathrm{Q}=5 \mathrm{GeV}$, and on bottom is $\mathrm{Q}=100 \mathrm{GeV}$. These are the examples that are consistent with DIS+Drell-Yan data sets (see text). 


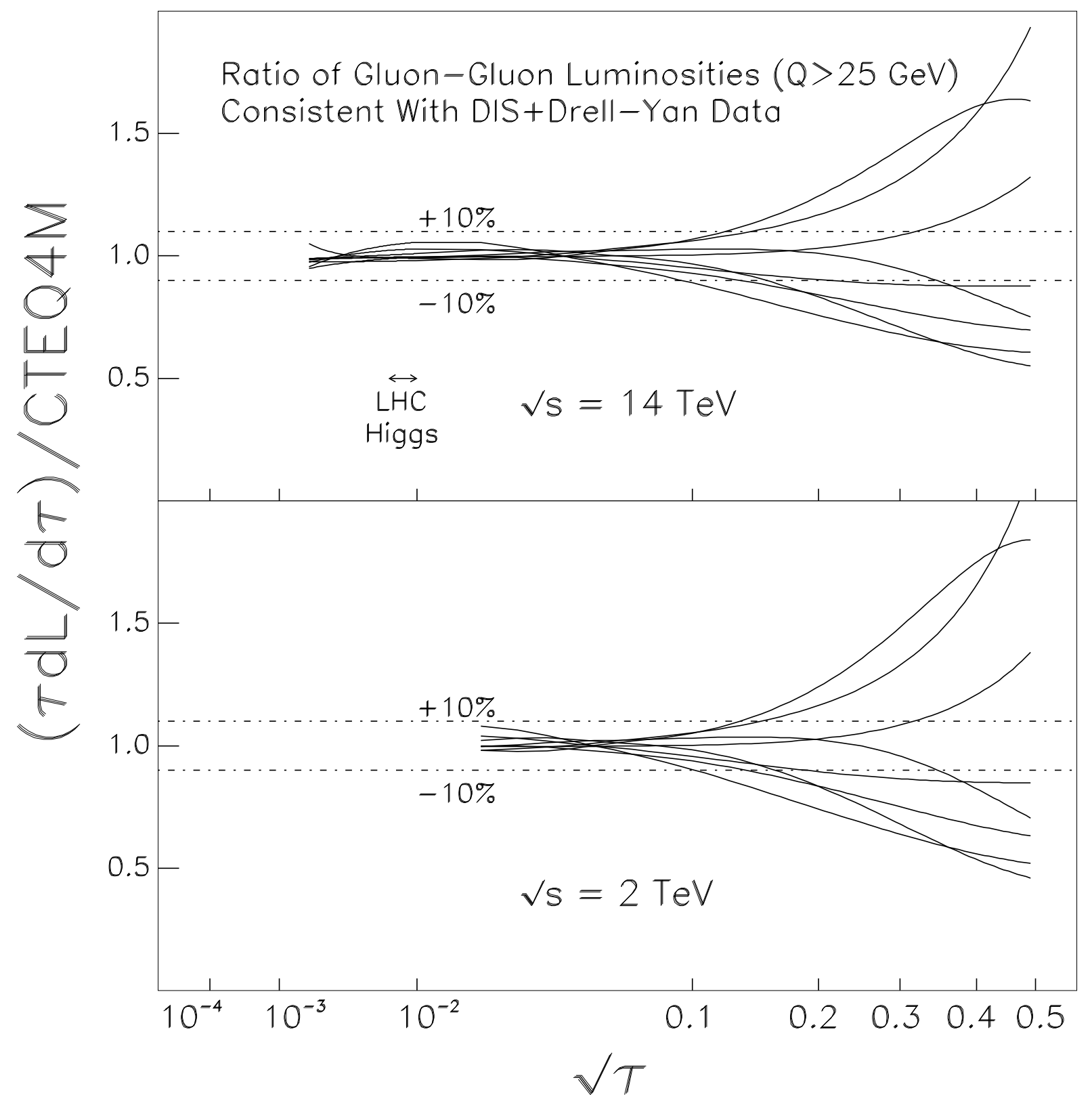

Figure 6: The ratio of integrated gluon-gluon luminosities compared to CTEQ4M is shown as a function of $\sqrt{\tau}$. These are the examples that are consistent with DIS+Drell-Yan data sets. 


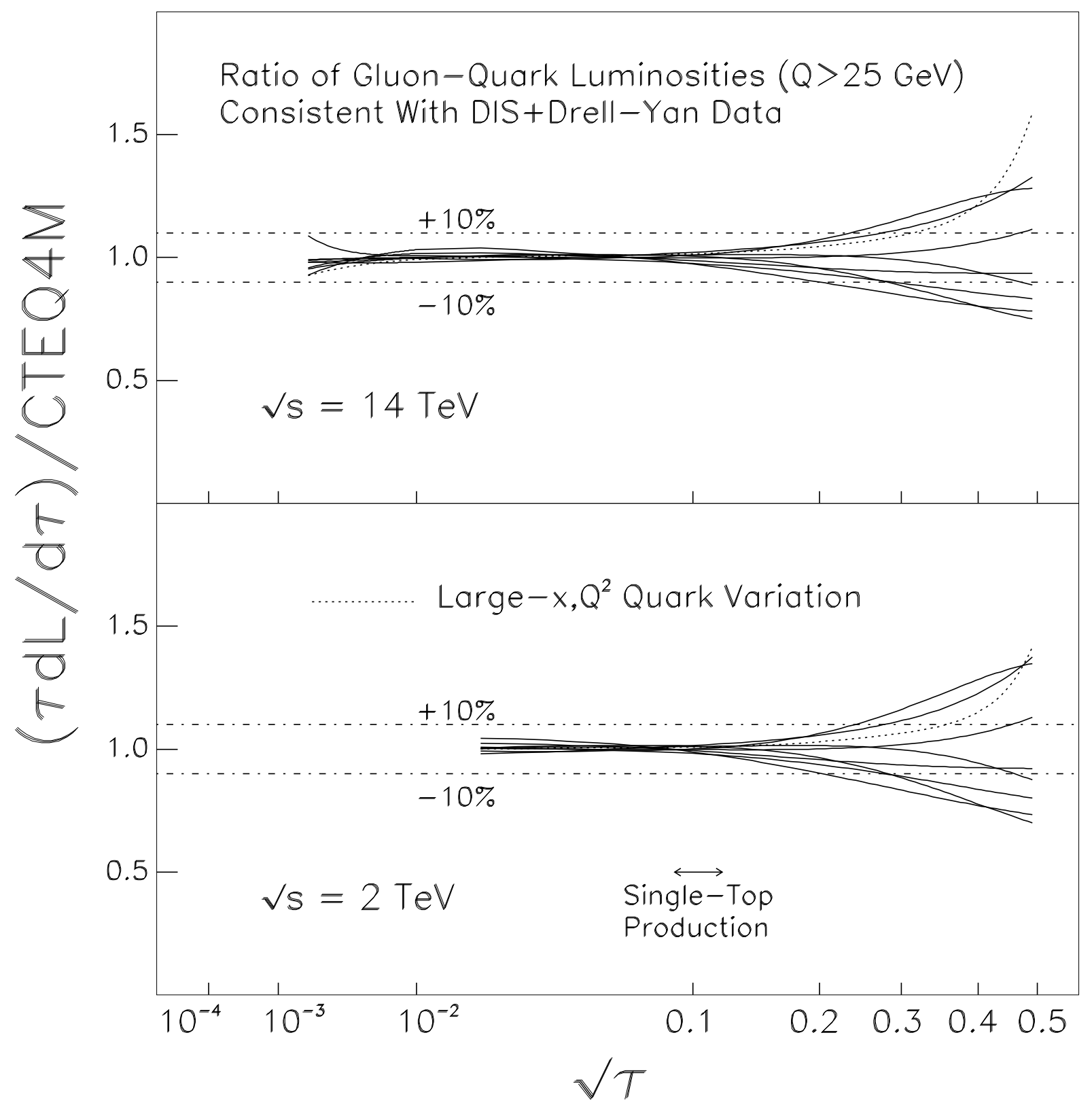

Figure 7: The ratio of integrated gluon-quark luminosities compared to CTEQ4M is shown as a function of $\sqrt{\tau}$. These are the examples that are consistent with DIS+Drell-Yan data sets (see text). 